\title{
ELEMENTOS PARA LA COMPOSICIÓN DE LOS SISTEMAS JURÍDICOS MAESTROS*
}

\author{
Jorge Enrique León Molina \\ Universidad Católica de Colombia
}

\section{Resumen}

En este artículo se pretende mostrar unas definiciones respecto al sistema jurídico maestro, su enunciación en el marco de una teoría general de los sistemas y su ubicación como un sistema operativamente clausurado a la luz de la teoría de los sistemas de Niklas Luhmann, para determinar una formulación lógica del entorno que soporte ese sistema y así construir el sistema bajo dos bases: la completitud y la consistencia, desde la idea de la división de poderes.

Para logar el objetivo propuesto, se dividirá el texto en seis secciones, a partir de los elementos de los distintos sistemas jurídicos y finalizará con el tema de la consistencia dentro de los sistemas jurídicos.

Palabras clave: lenguaje, acción, norma, ley.

El autor: abogado. Docente e investigador del Grupo de Estudios Legales y Sociales "Phronesis" adscrito al Centro de Investigaciones Socio Jurídicas (Cisjuc), Facultad de Derecho, Universidad Católica de Colombia. Dirección postal: diagonal 141 A No. 110A-35 bloque 31 apartamento 201. Correo electrónico: leon_0904@hotmail.com y jeleon@ucatolica.edu.co

Recibido: 15 de marzo de 2013; evaluado: 19 de abril de 2013; aceptado: 15 de mayo de 2013.

* Este artículo forma parte del trabajo de investigación realizado por el autor en el grupo de estudios legales y sociales "Phronesis", adscrito al Centro de Investigaciones de la Universidad Católica de Colombia (Cisjuc). 


\title{
ELEMENTS TO COMPOSE THE MAIN LEGAL SYSTEMS*
}

\author{
Jorge Enrique León Molina \\ Universidad Católica de Colombia
}

\begin{abstract}
This article means to present some definitions regarding the main legal system, its enunciation in the framework of a general systems theory and its situation as a system that has been operationally ended in the light of Niklas Luhmann systems theory, to determine a logical formulation of the context that supports that system and thus build it on the basis of completeness and consistency from the idea of separation of powers.
\end{abstract}

To achieve our goal, the text is divided in six sections, beginning with the elements of the different legal systems and ending with the issue of consistency in them.

Keywords: language, action, norm, law.

About the author: Lawyer. Research professor attached to the Grupo de Estudios Legales y Sociales Phronesis (Legal and Social Studies Research Group Phronesis), from the Centro de Investigaciones Socio-Jurídicas (Center for Socio-Legal Research) of the Faculty of Law at the Universidad Católica de Colombia. Address: Diagonal 141 A No. 110A-35 bloque 31 apartamento 201, Bogotá, Colombia. E-mail: león_0904@hotmail.com and jeleon@ucatolica.edu.co

Received: March 15, 2013; reviewed: April 19, 2013; accepted: May 15, 2013.

* This article is part of the research work by the author on the Grupo de Estudios Legales y Sociales Phronesis (Legal and Social Studies Research Group Phronesis), from the Centro de Investigaciones Socio-Jurídicas (Center for Socio-Legal Research) of the Faculty of Law at the Universidad Católica de Colombia. 


\section{Introducción}

A la hora de hacer reconstrucciones de sistemas jurídicos maestros, es menester determinar la pertinencia de su estudio de forma lógica y filosófica, para así demostrar sus relaciones operativas. Por esta razón, se hace necesario definir los elementos que puedan llegar a componer ese sistema a reconstruir, con base en la idea de que todo sistema jurídico normativo debe estar justificado en una estructura tajante de separación de poderes. Luego, se tratará de señalar la composición lógica del entorno que soporta ese sistema, sus componentes principales y su relación en una formulación que permita plena armonía entre sí y con el sistema a reconstruir. En tercer lugar, se harán ciertas precisiones acerca del sistema que se ha de construir, a la luz de la teoría de los sistemas de Niklas Luhmann, en procura de la expresión de una idea de concordancia lógico-sociológica del mismo. En cuarto lugar, se planteará una idea de los sistemas jurídicos maestros completos, es decir, aquellos que requieren que su corpus normativo ${ }^{1}$ cubra todas las necesidades fácticas de dicho sistema o, mejor, del entorno que lo soporta. En sexto lugar, se propondrá una idea de consistencia, en virtud de la cual cada acción del sistema debe estar en concordancia con las demás acciones del sistema; se explica, en este caso, ciertos asuntos de inconsistencia desde la teoría del Derecho de Nino y de Ross. El artículo finaliza con una breve explicación de los contenidos semánticos de las proposiciones que compondrían este hipotético sistema.

\section{Elementos de una idea de sistema normativo}

Respecto a los elementos propios del sistema normativo, en las teorías de justificación de los sistemas jurídicos existen diversas formas de distinguir entre fuentes formales y fuentes materiales del Derecho. Alchourrón ${ }^{2}$ considera una versión más estricta de la diferenciación de fuentes, que se explica a continuación:

\subsection{Formales}

La legislación es la fuente formal del Derecho por excelencia. La costumbre, así como otro tipo de normas, es tenida en cuenta solo cuando la ley lo indica.

Georg Henrik Von Wright, Practical Reason. Vol. 1 (Denver: Cornell University Press, 1983), 44.

Carlos Alchourrón, Fundamentos para una teoría general de los deberes (Madrid: Marcial Pons S. A., 2010), 165. 


\subsection{Materiales}

Las fuentes materiales del Derecho, según este enfoque, son las siguientes:

- La moral. No se discute la naturaleza formal o material de esta fuente, pero se afirma que es material.

- La jurisprudencia. Se justifica el hecho de que la jurisprudencia no es una fuente formal del Derecho, por cuanto, si una decisión judicial deriva de las leyes del Estado, la jurisprudencia no le aporta nada a dichas leyes y, si no se tiene en cuenta la ley en la jurisprudencia, se viola el principio de legalidad.

La anterior distinción deriva de la doctrina de separación de poderes y se enfoca en la separación del poder legislativo del judicial, así:

- Legislativo. Citando la doctrina de separación de poderes, es la principal autoridad competente para crear las normas que sirven de referente de la conducta de los individuos en sociedad.

- Judicial. Consiste en resolver los casos particulares, de acuerdo con las normas emanadas del legislador.

Dicha separación de poderes, a su vez, se justifica en tres argumentos, que son:

- El argumento de la igualdad. Consiste en que, cuando los jueces aplican las leyes emanadas del legislativo, todos los casos del mismo tipo recibirán la misma solución. En consecuencia, la exigencia del ideal político de igualdad justifica también la separación de poderes.

- El argumento de la democracia. En aquellos países en donde se eligen popularmente los miembros del legislativo, no ocurre lo mismo con los miembros de la rama judicial, por lo cual, una separación tajante de esos poderes permite el control democrático sobre el modo de resolver los conflictos de los ciudadanos.

- El argumento de la certeza y la seguridad. Solo mediante una "estricta" división de poderes la persona está en condición de conocer sus derechos y sus obligaciones, antes de que haya sentencia judicial que los enuncie. Esta es la 
razón de la justificación de las sentencias judiciales, ya que, si no se hace, esta sería arbitraria y los derechos y obligaciones serían establecidos por el juez.

Aun con una separación tajante y radical de poderes, los resultados obtenidos no garantizan las cualidades intrínsecas de las decisiones adoptadas; entonces, en este modelo del sistema maestro, la justicia en las decisiones judiciales deviene de las normas generales emanadas del Legislativo, cerrando toda opción a la equidad de obtener un lugar en el sistema maestro.

\section{Mundos posibles y estructuras de modelos}

Para Alchourrón, todo sistema jurídico maestro debe estar inmerso en una realidad determinada, que no es más que una estructura de modelo que permita la creación del sistema jurídico maestro L determinado y obedece a la siguiente forma lingüística:

$$
\mathrm{Ra}=<\mathrm{C},{ }^{*}, \mathrm{~A}, \mathrm{~F}_{\mathrm{o}}^{\alpha}, \mathrm{F}_{\mathrm{p}}^{\alpha}>
$$

Se compone de los siguientes elementos:

$\mathrm{C}=$ conjunto de circunstancias posibles, es decir, de circunstancias fácticas presentes en esa realidad.

* = elemento de C, que representa el momento actual de una circunstancia fáctica posible, del siguiente modo:

$$
(* \in C)
$$

Donde * expresa la pertenencia de la acción en un tiempo determinado.

$A=$ representa las acciones posibles en un tiempo definido, sobre una determinada circunstancia fáctica.

$\mathrm{F}^{\alpha}{ }_{\mathrm{o}}=$ representa una serie de proposiciones de acción que constituyan conjuntos modelos que recaen en $\mathrm{A}^{3}$

- $\quad$ Esta presentación axiomática de la realidad permite la construcción de modelos específicos de sistemas jurídicos soportados en ella misma, en la medida en

\footnotetext{
Ulises Schmill, Lógica y Derecho. 2a ed. (México D. F.: Fontamara, 1997), 59.
} 
que la aproximación semántica que se obtenga de esa realidad axiomatizada posibilite justificar una idea intuitiva de la realidad subyacente a un sistema jurídico. Si esta construcción intuitiva del sistema jurídico maestro parte de una formulación axiomática de la realidad, ${ }^{4}$ a la hora de construir tal sistema maestro representan un determinado conjunto de axiomas que permiten expresar tanto la completitud, como la consistencia interna de dicho sistema maestro, atendiendo a que la base intuitiva del sistema jurídico a construir define:

- La obligatoriedad de un conjunto de acciones determinadas.

- Que ordenar una acción no implica su necesaria autorización.

- Que la permisión de una acción está íntimamente implicada con una acción autorizada por dicho sistema.

Se estaría partiendo de la base de toda construcción de sistemas, es decir, de una realidad que soporta tal sistema mediante unas bases intuitivas que expresen el porqué de su conformación; en otras palabras, la justificación del sistema es la intuición de su utilidad.

\section{Sistema según Niklas Luhmann}

Para Luhmann, el sistema es considerado como un conjunto de operaciones de carácter fáctico que, al ser compuestos de operaciones sociales, son constituidas como comunicaciones, cuyo "punto de partida para su definición es, sin lugar a duda, la distinción entre sistema/entorno y no la norma, o una determinada tipología de valores". 5

Es importante tener en cuenta el concepto de operación dentro del sistema: según Luhmann, es el acontecimiento que constituye una determinada actualización de posibilidades fácticas que, en cuanto suceden, desaparecen. No posee un tiempo definido dentro del sistema de la sociedad o, dicho de otro modo, un término de duración determinado, a menos que sea para su observación dentro del sistema; en ese caso, sí tiene un tiempo o un término determinado (pasado, presente o

Alchourrón, Fundamentos para una teoría general de los deberes, 84.

Niklas Luhmann, El derecho de la sociedad (México D. F: Herder, 2005), 98. 
futuro). En otras palabras, las operaciones son hechos que se presentan al interior del sistema en un tiempo presente determinado.

De acuerdo con Luhmann, el sistema de Derecho, es decir, "todo sistema que tiene como función la emanación de normas jurídicas, que constituyen soluciones a casos emergentes", ${ }^{6}$ conocido también como sistema jurídico (en adelante, entendido como sistema jurídico maestro), constituye un sistema cerrado, por cuanto se observa y se describe a sí mismo, de forma que desarrolla sus propias teorías, comportándose de una forma constructivista, que no intenta representar el mundo exterior —o sea, el entorno del sistema—, sino construirse de acuerdo con su propia información.

En concordancia con lo anterior, es importante hacer una distinción entre el sistema jurídico y el entorno que lo soporta, a fin de mostrar el origen cierto de los sistemas jurídicos y sus relaciones fácticas con el ambiente en donde se desarrollan. Así, luego de hacer un estudio histórico de la diferencia entre estos dos términos, Luhmann desarrolla dos concepciones para la misma: la sociológica y la jurídica.

\subsection{Concepción sociológica}

Para la Sociología, según Luhmann, toda esta distinción entre sistema y entorno obedece a un problema de interdependencia entre estos conceptos, a fin de que se pudiese representar a la sociedad (el entorno del sistema) como un factum, ${ }^{7}$ es decir, un elemento productor de normas, dirigido con la finalidad de orientar los diversos sistemas presentes en él.

Es imposible describir hechos sociológicos con base en la distinción norma/hechos, porque el objeto de estudio de la Sociología no es el sistema jurídico, sino el sistema de la ciencia y estos conceptos son indiferentes a él.

\subsection{Concepción jurídica}

Para Luhmann, es el sistema del Derecho —el sistema jurídico, en nuestro caso—el que plantea las distinciones y descripciones que utiliza en sus operaciones. Por lo tanto, la unidad del Derecho al interior del sistema jurídico se basa en su autoproducción, denominada "autopoiesis". ${ }^{8}$ Entonces, la sociedad es considerada como

Luhmann, El derecho de la sociedad, 77.

Luhmann, El derecho de la sociedad, 84

Luhmann, El derecho de la sociedad, 85 
un determinado orden social que tiene la función de soportar y hacer posible la función integradora del Derecho.

Para reforzar la idea del sistema jurídico maestro como un sistema cerrado, Luhmann expresa que el sistema tiene la facultad de verificarse a sí mismo y, mientras no lo haga, no produce consecuencias jurídicas en su entorno.

Cuando aparecen elementos al interior del sistema, denominados "extrajurídicos", también surgen confirmados en las normas positivas del sistema, con más exactitud, en las normas constitucionales del mismo; esto le otorga el carácter de reconocible a estos elementos extrajurídicos al interior del sistema.

Dentro de este contexto, la norma jurídica debe ser entendida como "una forma específica de expectativa fáctica", que se puede observar de dos formas:

- Psíquicamente, es decir, cuando se sabe qué se quiere expresar en esa norma.

- Mediante el sentido que ella (valga decir, la norma) misma expresa, que es comprendida por el intérprete gracias a la comunicación.

Como dichas expectativas existen o no, en el evento en que deban existir, no hay necesidad de producir un deber ser normativo, sino que, en el plano de las expectativas, se puede anticipar normativamente lo que se puede esperar del plano de la realidad fáctica.

La teoría de sistema de Luhmann parte de la idea de que el sistema jurídico es un subsistema del sistema de la sociedad; por lo tanto, no interesa (en principio) la influencia de la sociedad sobre el Derecho, por cuanto la unidad del sistema jurídico se produce y se reproduce única y exclusivamente por el sistema jurídico mismo y no por medio de los factores presentes en el entorno que intenten incidir en él.

Entonces se afirma que las relaciones entre el sistema jurídico, como subsistema del sistema de la sociedad, son ambiguas, porque:

- La sociedad constituye el entorno del Derecho, es decir, del sistema jurídico.

- Todas las operaciones del sistema jurídico se realizan en el seno de la sociedad, o sea, en su interior. Esto quiere decir que dichas operaciones son tanto jurídicas, como sociales. 
Como lo explica Luhmann, el sistema jurídico desarrolla sus operaciones "jurídicas", las cuales son también sociales, creando un entorno específico para la existencia de dicho sistema jurídico dentro del sistema de la sociedad.

La unidad del sistema jurídico maestro dentro de la sociedad — sus límites y sus funciones - se produce y se reproduce mediante operaciones propias de dicho sistema, denominada "cláusula operativa" y dichas operaciones no son otra cosa que la comunicación plena de sentido, es decir, que el sistema jurídico maestro no hace otra cosa que crear formas lingüísticas, en forma de oraciones, que se intentan dar a conocer al sistema social por medio de la comunicación, respetando los límites que el mismo sistema de la sociedad traza para ello.

Este sistema maestro está diseñado para satisfacer los ideales políticos de seguridad e igualdad formal, pero, difícilmente garantizaría otros ideales como la justicia o la equidad, aunque uno de los propósitos del sistema maestro sea optimizar el servicio de justicia. El sistema maestro debe satisfacer dos condiciones para ofrecer la seguridad y la objetividad que necesita en este punto. Son las condiciones de completitud y consistencia.

\section{Completitud}

Este postulado exige que el sistema tenga u ofrezca una solución para todo caso, aunque esto es imposible, según ciertos críticos. Por lo tanto, es necesario dilucidar las concepciones de caso para entender esta afirmación.

Por ejemplo, cuando se habla de homicidio, se alude a una mera descripción de ciertas propiedades insertas dentro de unos acontecimientos, ya que, de por sí, "homicidio" como tal solo expresa una acción sobre un sujeto determinado (político, simple, agravado, etcétera.), lo que hace que esta expresión —homicidio- sea ambigua.

Para entender el sentido de este postulado se tiene que eliminar esta ambigüedad. Alchourrón introduce los términos caso genérico y caso individual, que se aplican para:

- Cada acontecimiento concreto que posea una propiedad común es un caso individual. 
- Para la clase de acontecimientos que posean una propiedad común se usa el término caso genérico.

Se afirma que este postulado de completitud está vinculado con los casos genéricos, pero, como esta noción impone diversas exigencias en distintos contextos, la estructura de este postulado es la siguiente:

- Para un determinado problema jurídico, las normas del sistema seleccionan un grupo de propiedades como relevantes.

- Según la presencia o ausencia de cada una de las propiedades relevantes se puede construir un conjunto de casos genéricos elementales.

Los casos jurídicos elementales son aquellos en los que cada una de sus propiedades relevantes está ausente o está presente.

Los dos anteriores presupuestos de la estructura del sistema maestro permiten inferir que:

- Cuando para cada uno de los casos jurídicos elementales hay una norma en el sistema que establece la solución para ese problema jurídico, el sistema es completo.

- Cuando ninguna norma en el sistema indica cómo resolver el problema jurídico en cuestión en un solo caso genérico elemental, se enfrenta a una laguna normativa en el sistema.

Todo esto nos permite afirmar que cada caso individual debe pertenecer solo a un caso genérico elemental. En este sentido, un sistema completo que brinda las respuestas a todos los casos genéricos elementales también brinda, de manera indirecta, las soluciones normativas para todos los casos individuales que se presenten en el sistema.

También se infiere que una laguna en el sistema es normativa, porque surgen de la ausencia en dicho sistema de una norma de carácter general que permita dar consecuencias normativas para un caso genérico. Por lo tanto, según Alchourrón, la única forma de superar una laguna normativa es ampliando el sistema, es decir, incorporando en él una nueva norma para resolver ese caso que no estaba previsto: 
"Es importante aclarar que, tanto la Completitud como la Consistencia del sistema, son relativos a un conjunto de normas, y no a un conjunto de Casos Genéricos".

\section{Consistencia}

Este postulado exige que no existan soluciones normativas incompatibles con el conjunto de normas positivas aplicables a casos genéricos.

La consistencia tiene una característica especial y es que, en Alchourrón, ${ }^{10}$ se trata de "una propiedad de los significados atribuidos a los textos normativos y no de los textos normativos mismos". ${ }^{11}$

Los anteriores enunciados tratan sobre las relaciones entre normas dentro de un sistema maestro. Teniendo en cuenta que el Sistema Maestro es un conjunto de normas y no un conjunto de expresiones lingüísticas. Así, norma es el significado que se le atribuye a una expresión lingüística y no la propia expresión. Como las expresiones de la norma —códigos, leyes, Constituciones- son entidades lingüísticas, forman parte del sistema, pero están contenidas dentro de lo que se denomina Libro Maestro.

Esto quiere decir que, en caso de que un texto legal reciba dos o más interpretaciones diferentes entre sí, sustentadas en normas jurídicas que sistemáticamente están contenidas en un libro maestro, entendiendo este último como el orden sistémico de normas, es decir, de entidades lingüísticas inicialmente interpretadas.

\subsection{Inconsistencias desde Carlos Santiago Nino}

Nino expresa un concepto de inconsistencia basado en el concepto de contradicción entre las normas, que surge cuando dos normas imputan, al mismo caso, soluciones incompatibles entre sí. ${ }^{12}$ Un sistema es inconsistente cuando para cada caso existen dos o más soluciones, de modo que la conjunción de tales soluciones sea contradictoria.

Según este orden de ideas, existen dos condiciones para que se presente el fenómeno de la inconsistencia normativa:

\footnotetext{
Alchourrón, Fundamentos para una teoría general de los deberes, 167.

Alchourrón, Fundamentos para una teoría general de los deberes, 167.

Alchourrón, Fundamentos para una teoría general de los deberes, 167

Carlos Santiago Nino, Introducción al análisis del Derecho (Barcelona: Ariel S. A., 1983), 273.
} 
- Que las normas tengan el mismo ámbito de aplicabilidad, o sea, que el mismo caso tenga dos o más normas al cual se refieran. En otras palabras, que tengan las mismas circunstancias fácticas. Esto puede ocurrir por tres razones:

- La descripción de un caso que hace una norma es equivalente a la descripción que hace otra norma.

- Una de las descripciones implica lógicamente a la otra descripción.

- A pesar que las descripciones son independientes, existen casos que pueden ser descritos por ambas normas.

- Que las normas (o el sistema jurídico maestro) impute a un caso determinado soluciones lógicamente incompatibles entre sí.

Es importante aclarar que, para calificarse como incompatibles, las soluciones emanadas de las normas o del sistema dependen de la lógica deóntica que se presuponga en dicho sistema.

Nino presenta una figura en la que se muestran las relaciones de Compatibilidad (-) e Incompatibilidad (/) entre los operadores deónticos, Facultad (F), Permisión (P), Prohibición (Ph), y Obligación (O) de la siguiente forma:

Figura 1. Relaciones de compatibilidad

\begin{tabular}{ccc}
\hline$F$ & - & $P$ \\
\hline$F$ & $/$ & $P h$ \\
\hline$F$ & $/$ & 0 \\
\hline$P$ & - & 0 \\
\hline$P$ & $/$ & $P h$ \\
\hline$P h$ & $/$ & 0 \\
\hline
\end{tabular}

Fuente: Nino ${ }^{13}$

13 Nino, Introducción al análisis del Derecho, 275. 
Existe otra clasificación, que se analiza a continuación.

\subsection{Inconsistencias según Alf Ross}

Independientemente del tipo de relación que puede haber entre las descripciones normativas con soluciones incompatibles, existe también un tipo de clasificación que se hace de acuerdo con el grado de superposición de las descripciones normativas. En palabras de Ross, se presenta la inconsistencia entre normas "cuando estas imputan efectos jurídicos incompatibles a las circunstancias de hecho que quieren analizar; creando asî conflictos normativos en un momento determinado a razón del ejercicio del derecho". ${ }^{14}$ Es en este punto donde Alf Ross distingue tres clases de inconsistencias:

\subsubsection{Inconsistencia total-total}

Esta inconsistencia se presenta cuando los ámbitos de referencia de ambas normas se superponen por completo, es decir, cuando la situación requerida en una norma encaja a la perfección en la situación descrita en otra norma contradictoria lógicamente. Se expresa de la siguiente forma:

Figura 2. Inconsistencia total-total

\section{Situación descrita en la Norma B}

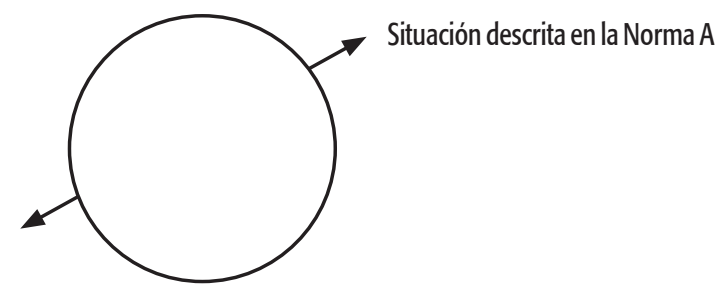

Fuente: Ross ${ }^{15}$

Ejemplo: cuando una norma A define una multa para quien aparque en una bahía y una norma B expresa la creación de más bahías con fines de aparcamiento.

\subsubsection{Inconsistencia total-parcial}

Esta inconsistencia surge cuando, en los ámbitos de referencia de dos normas, una está incluida en otra, pero la última comprende, además, varios casos adicionales que se pueden llegar a presentar. Se puede graficar de la siguiente forma:

14 Alf Ross, Sobre el Derecho y la justicia (Buenos Aires: Editorial Universitaria de Buenos Aires S. E. M., 1963), 242.

15 Ross, Sobre el Derecho y la justicia, 242. 
Figura 3. Inconsistencia total-parcial

Situación descrita en la Norma B

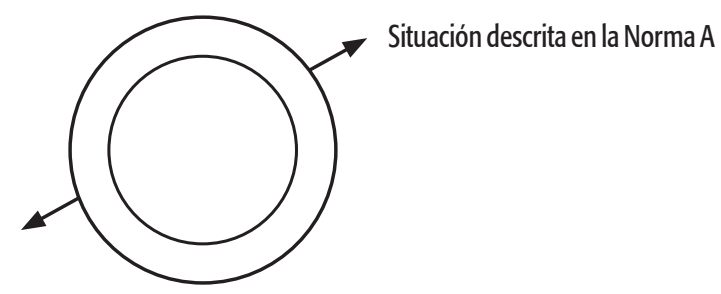

Fuente: Ross ${ }^{16}$

Ejemplo: cuando una norma A indica que la Fiscalía deberá acusar frente a la jurisdicción penal a quienes cometan todo tipo de delitos y la norma B señala que en los delitos denominados pequeñas causas, por ser de poca monta, los acusados sean llevados a la jurisdicción penal por abogados o por estudiantes de Derecho.

\subsubsection{Inconsistencia parcial-parcial}

Esta inconsistencia aparece cuando los ámbitos de referencia de dos normas son autónomos, mas la descripción de las normas, cuya solución es incompatible lógicamente entre sí, se superpone de manera parcial. Se expresan así:

Figura 4. Inconsistencia parcial-parcial

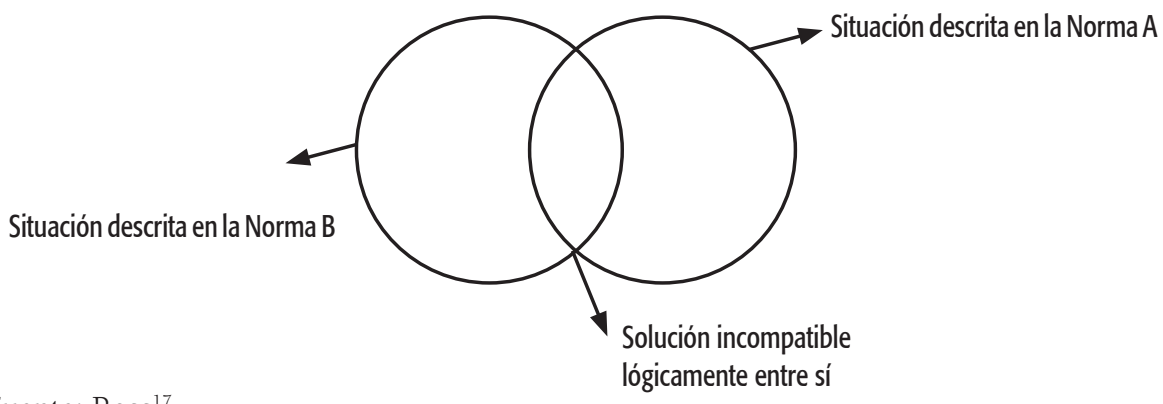

Fuente: Ross ${ }^{17}$

Ejemplo: cuando una norma A determina que quienes posean automóviles deben pagar impuestos y la norma B establece que los equipos de construcción están

6 Ross, Sobre el Derecho y la justicia, 244.

17 Ross, Sobre el Derecho y la justicia, 245. 
exentos de pagar impuestos. Así, están en discusión las volquetas, excavadoras y demás que, según A son vehículos, pero según B están exentos de pagar impuestos.

Estas inconsistencias pueden ser salvadas de acuerdo con unas determinadas reglas para la resolución de los problemas de contradicción e inconsistencia normativa. A juicio de Alf Ross ${ }^{18}$ los juristas y los jueces emplean varias reglas tendientes a resolver los problemas de contradicción normativa y, por consiguiente, de inconsistencia, constituidas por tres principios: principio lex superior, principio lex posterior y principio lex specialis, que, según Ross, determinan la anulación de dichos problemas sistémicos.

- Principio lex superior

Este principio nos indica que, entre dos normas que sean contradictorias, con diversa jerarquía, debe prevalecer siempre la que tenga un orden superior, es decir, más jerárquica que su contradictoria.

Por ejemplo, es bien sabida la prevalencia de la norma constitucional sobre las demás normas del sistema jurídico maestro.

Este principio es esencial para que funcione la delegación de poderes dentro del sistema, pero posee muchas excepciones, por ejemplo, cuando hay dos normas contradictorias con igual posición jerárquica en el sistema.

- Principio lex posterior

Este principio nos expresa que la norma más reciente prevalece sobre la que fue promulgada con anterioridad. Este principio tiene un ámbito de aplicación general dentro del sistema, por cuanto justifica el fenómeno jurídico de la derogación de normas, aunque entra en conflicto cuando se intenta aplicar sobre una norma superior.

- Principio lex specialis

Este principio nos indica que se debe dar preferencia a la norma específica que está en conflicto, que a aquella cuyo campo de aplicación sea más general. Al igual que

18 Nino, Introducción al análisis del Derecho, 275. 
las anteriores, esta regla está en conflicto cuando la norma general es superior o cuando la norma general es posterior a la norma particular.

Estos principios resultan de la pretensión de los operadores jurídicos de solucionar de modo mecánico las inconsistencias al interior de todo sistema jurídico maestro. Cabe aclarar que estos no constituyen leyes lógicas, sino evaluaciones pragmáticas que dan lugar a excepciones irregulares y no dan solución satisfactoria al problema de la inconsistencia, sobre todo por:

- No tener reglas de segundo orden que solucionen los conflictos entre estos principios.

- Casos de contradicciones normativas en normas con igual jerarquía, normas que fueron promulgadas simultáneamente o normas que tienen el mismo grado de generalidad.

Para identificar un sistema maestro, se debe trabajar con una interpretación del Libro Maestro y, en este caso, se puede estudiar su consistencia y su completitud. Dichas interpretaciones del sistema maestro deberían tener una "referencia empírica", según Alchourrón, ${ }^{19}$ por cuanto los significados atribuidos a sus componentes lingüísticos deben referirse a situaciones de la vida cotidiana. Este hecho, a pesar de ser una condición para aplicar las normas generales del sistema maestro, también genera la mayoría de las incertidumbres que se deben superar para llegar al modelo ideal. ${ }^{20}$

Desde el punto de vista teórico, el sistema maestro es el ideal de la máxima objetividad, a pesar de basarse en presupuestos cuasi irreales, derivados de las dificultades interpretativas que surgen a la hora de revisar el Libro Maestro. El impacto que tiene dicho sistema ideal, denominado sistema maestro, se puede detectar en cada sistema jurídico contemporáneo, con base en tres principios del sistema maestro en relación con su concepción. Estos son:

- Inexcusabilidad. Este principio consiste en que los jueces deben resolver todos los casos que se sometan a juzgamiento dentro de la esfera de su competencia. Este principio tiene, a su vez, tres características:

\footnotetext{
19 Alchourrón, Fundamentos para una teoría general de los deberes, 156.

20 Alchourrón, Fundamentos para una teoría general de los deberes, 157.
} 
- Dicha exigencia en el Derecho Positivo aparece muchas veces en forma de una prohibición de abstenerse de dictar fallo.

- Este principio supone que la competencia de los jueces es exhaustiva, ya que no pueden oponerse a fallar.

- Para cada caso existe un juez con competencia y con la obligación de fallar.

- Justificación. Consiste en que toda decisión judicial requiere una justificación, un fundamento o una razón, por lo que todos los jueces están en la obligación de exponer las razones de sus decisiones. Además, se exige al juez que su decisión no sea arbitraria, lo cual se previene motivando las sentencias judiciales.

Este principio tiene una finalidad que, según Alchourrón, es "eliminar las posibles fuentes de injusticias que se puedan incorporar a las decisiones judiciales que carecen de razón suficiente", ${ }^{21}$ es decir, de una justificación.

En general, este principio se encuentra incluido en forma de códigos de procedimiento como reglas o normas, como obligaciones impuestas a los jueces.

- Legalidad. Este principio expresa que las decisiones judiciales deben estar fundadas en normas jurídicas del Estado y permite suponer que los fundamentos requeridos en toda providencia deben ser jurídicos, limitando la clase de enunciados que se puedan emplear como fundamentos admisibles o fundamentos jurídicos válidos.

Los anteriores principios pueden resumirse en la siguiente frase: "Los Jueces deben resolver todos los casos que se le sometan a Juzgamiento dentro del límite de su competencia, mediante decisiones fundadas en normas jurídicas del Sistema". ${ }^{22}$

A la luz del enunciado anterior, estos principios no suponen una obligación imposible para el juez, ya que se impone que "en todo Sistema Jurídico hay normas que proveen fundamentos para resolver todo caso posible", ${ }^{23}$ creando el postulado

Carlos Alchourrón, "Derecho y lógica", Isonomía 3, núm. 13 (2000): 331-348.

Alchourrón, Fundamentos para una teoría general de los deberes, 158.

Alchourrón, Fundamentos para una teoría general de los deberes, 158. 
de la completitud o ausencia de lagunas, que se revisará más adelante. La verdad o falsedad de este enunciado depende de:

- Cómo interpretar la noción de justificación.

- Cómo identificar los elementos de un sistema jurídico.

Se plantea una versión ideal, en la que deben satisfacerse dos condiciones para proporcionar una justificación. Estas son:

- El contenido de una decisión judicial debe ser una consecuencia lógica de las premisas que la fundamentan, aunque hay una excepción única, que es: las reglas generales brindan al juez discreción para tomar la decisión dentro de ciertos límites.

- Las premisas normativas empleadas en la justificación deben ser generales.

Alchourrón compara la justificación —incluso encuentra similitudes- con la teoría de la explicación de hechos particulares en las ciencias empíricas, de Carl G. Hempel. ${ }^{24}$ De acuerdo con esta teoría, un hecho empírico es una deducción lógica, cuya consecuencia es:

- Una descripción del hecho a explicar, llamado explanandum.

- Premisas, llamadas explanans.

Por lo tanto, son enunciados de dos clases:

- Conjunto de leyes generales.

- Conjunto de enunciados individuales, que describen las condiciones iniciales antecedentes.

Según esta teoría, toda explicación debe satisfacer diversas condiciones de adecuación, que son:

24 Georges Kalinowsky, Lógica del discurso normativo (Madrid: Tecnos, 1975), 45. 
- El explanandum debe ser lógicamente implicado por el explanans, es decir, la descripción del hecho a explicar debe ir dentro de premisas.

- El explanans, o sea, la premisa usada para explicar, debe contener leyes de carácter general.

- Las oraciones que constituyen el explanandum deben ser verdaderas (conocidas como tautologías normativas). Los hechos a explicar debieron haber ocurrido en el mundo real. ${ }^{25}$

De acuerdo con esta teoría, las leyes generales brindan el componente explicativo básico que debe contener toda explicación y la lógica, en este evento, asume la función de vincular el contenido conceptual de estas leyes con lo que ocurre en el mundo real. Dicho de otro modo, el explanans está dentro del explanandum y la lógica se encarga de vincularlas y asociarlas con el mundo real.

La tesis empírica utilizada se justifica en el hecho de que, a pesar de que para las ciencias empíricas no haya explicación para cada hecho, el principio de causalidad universal ${ }^{26}$ que es una versión del principio de razón suficiente, constituye el principio normativo de completitud.

\section{Conclusiones}

En el marco de la reconstrucción de los sistemas jurídicos maestros, la completitud y la consistencia son criterios que se encuentran inmersos en medio de la composición interna de dicho sistema, como condiciones para el estudio de las operaciones lógicas internas en el sistema.

Las conclusiones que podríamos sacar del presente artículo son las siguientes:

En primer lugar, es necesario partir de la determinación de un entorno que soporte la idea de la reconstrucción de un sistema jurídico maestro, dado que este entorno arroja las primeras intuiciones respecto a la consistencia del sistema a reconstruir.

Richard Brevan Braithwaite, La explicación científica (Madrid: Tecnos S. A., 1965), 35.

26 Ludwig Von Bertalanffy, Teoría general de los sistemas. 4a ed. (Bogotá D. C.: Fondo de Cultura Económica, 1980), 47 . 
En segundo lugar, se evidencia que la autorreferencia del sistema obedece a un criterio fundamental a la hora de determinar la completitud del mismo, pues, cuanto más completo, más operativo.

En tercer lugar, la inconsistencia se erige como un problema lógico-semántico que amenaza con dotar al sistema reconstruido (el sistema jurídico maestro) de imprecisiones semánticas que, a la postre, obstaculizarán su operatividad en un momento determinado.

\section{Referencias}

Alchourrón, Carlos. "Derecho y lógica". Isonomía 13, núm. 13 (2000): 331-348.

Alchourrón, Carlos. Fundamentos para una teoría general de los deberes. Madrid: Marcial Pons S. A., 2010.

Braithwaite, Richard Brevan. La explicación cientifica. Madrid: Tecnos S. A., 1965.

Kalinowsky, Georges. Lógica del discurso normativo. Madrid: Tecnos, 1975.

Luhmann, Niklas. El derecho de la sociedad. México D. F.: Herder, 2005.

Nino, Carlos Santiago. Introducción al análisis del Derecho. Barcelona: Ariel S. A., 1983.

Ross, Alf. Sobre el Derecho y la justicia. Buenos Aires: Editorial Universitaria de Buenos Aires S. E. M., 1963.

Schmill, Ulises. Lógica y Derecho. México D. F: Fontamara, 1997.

Von Bertalanffy, Ludwig. Teoría general de los sistemas. 4a ed. Bogotá D. C.: Fondo de Cultura Económica, 1980.

Von Wright, Georg Henrik. Normas, verdad y lógica. México D. F: Fontana Mara, 2001.

Von Wright, Georg Henrik. Practical Reason. Vol. 1. Denver: Cornell University Press, 1983. 